\title{
A 3D Quantitative MRC Modeling Images Detected Case of Intrahepatic Biliary Stricture Diseases
}

\author{
Atsushi Nanashima ${ }^{a}$ Masanori Komi ${ }^{b}$ Marija Mavar ${ }^{c}$ Carlos Ferreira $^{c}$ \\ Paul O'Donoghue ${ }^{c}$ Marc Goldfinger ${ }^{c}$ Caitlin Langford ${ }^{c}$ \\ Naoya Imamura ${ }^{a}$ \\ aDivision of Hepato-Biliary-Pancreas Surgery, Department of Surgery, University of Miyazaki \\ Faculty of Medicine, Miyazaki, Japan; bivision of Radiology, Miyazaki University Hospital, \\ Miyazaki, Japan; 'Perspectum Ltd, Oxford, UK
}

\section{Keywords}

Intrahepatic biliary strictures · MR cholangiography · Quantitative imaging · 3D modeling

\begin{abstract}
In the future, the application of quantitative imaging and computational analysis will reduce the burden on radiologists. We herein report 8 pilot cases both with and without intrahepatic biliary stricture (IHBS) diseases which have been analyzed with the novel analytical system MRCP+ (Perspectum Ltd., Oxford, UK). The colored and well-visualized 3D models of the entire biliary trees could be obtained in all 8 cases. Three representative cases did not show dilated regions in the intrahepatic bile ducts. Cases diagnosed as a pancreatico-biliary maljunction showed slightly increased dilated visualization in the extrahepatic duct. Except in a case with severe stenosis resulting from hepatolithiasis, the number of visualized intrahepatic bile ducts tended to be decreased and the volume of biliary tree and the total length of stricture and dilatation were also decreased. However, the number of IHBS or dilatation was unchanged. The number of strictures obtained by MRCP+ and the subjective counts of stenosis from a radiologist was not found to be correlated. In a case of severe stenosis at the left lateral bile duct, the number of intrahepatic biliary dilatations was increased. The latest computerized 3D modeling technology was found to be useful in visualizing the alteration of intraluminal diameter of the entire biliary trees at a glance, which can provide the automatic diagnosis of IHBS diseases at an earlier phase.
\end{abstract}




\section{Introduction}

MRCP is increasingly becoming the preferred diagnostic modality for biliary diseases as it is noninvasive and offers similar rates of diagnostic accuracy to ERCP [1]. Despite this, the analysis of MRCPs is subjective and hence is hindered by inter-rater variability. In order to overcome this, a novel tool which models the biliary tree in 3D, yielding quantitative, repeatable and reproducible measures including diameters and volumes of intra- and extrahepatic bile ducts has been developed [2,3]. It is anticipated that such computational modeling of the biliary tree will give a more accurate representation of disease state than conventional MRCP analyses. Herein, we report the imaging profiles of 3 health volunteers and 5 patients with intrahepatic biliary diseases as obtained through both conventional and quantitative MRCP and consider the clinical diagnostic potential of the novel quantitative MRCP tool.

\section{Data Collection and Ethics}

We collected data from a prospectively maintained database of 5 patients with benign or malignant intrahepatic biliary diseases at Miyazaki University Hospital between October 2016 and July 2020. A further 3 patients with extrahepatic biliary diseases, without intrahepatic biliary abnormality, were also analyzed as a control in the same period. Histological diagnoses were determined for all patients, and all patients underwent surgical procedures. Patient demographics, clinicopathologic characteristics, and operative details were recorded. The study protocols followed the ethical principles of the Declaration of Helsinki 2008. This study protocol was reviewed and approved by the Clinical Research SupportCenter, University of Miyazaki, Faculty of Medicine approval number (\#0 - 0835, November 9, 2020, and \#0 799 , October 15, 2020). Informed consent for data collection was obtained by each patient, but no disclaimer was observed.

\section{MR Cholangiography}

MRCP imaging was carried out using either a Verio 3.0 T scanner (Siemens, Erlangen, Germany) or an Ingenia CX 3.0 T scanner (Philips, Eindhoven, Netherlands), with patients in the supine position under basal fasting conditions. Scanning conditions for the Verio $3.0 \mathrm{~T}$ scanner were as follows: an abdominal phased array coil was used: 3D fast spin echo with fat suppression (field of view $350 \mathrm{~mm}$, repetition time 2,000-5,000 ms, echo time $691 \mathrm{~ms}$, refocus angle 120 , acquisition matrix $240 \times 240$, recording matrix $320 \times 320$, receiver bandwidth 320 $\mathrm{Hz} / \mathrm{Px}$, recording slice thickness $1.1 \mathrm{~mm}$ ). Scanning conditions for the Ingenia CX $3.0 \mathrm{~T}$ scanner were as follows: an abdominal phased array coil was used as follows: 3D fast spin echo with fat suppression (field of view $320 \mathrm{~mm}$, repetition time 2,000-5,000 ms, echo time $600 \mathrm{~ms}$, refocus angle 120 , acquisition matrix $320 \times 256$, recording matrix $512 \times 512$, receiver bandwidth $296 \mathrm{~Hz} / \mathrm{Px}$, recording slice thickness $0.9 \mathrm{~mm}$ ). Oblique coronal sections were acquired in the planes of the common bile and pancreatic ducts with respiratory motion suppression techniques. A diagnosis was established by expert radiologists at the Department of Radiology, Miyazaki University Hospital. They assessed the shape of both intra- and extrahepatic bile ducts, and the symptom profiles were compared with the imaging findings. The raw DICOM MR imaging data without personal information was analyzed. Five of 19 cases (26\%) could be processed by Perspectum Ltd.'s quantitative modeling software, MRCP+. The high failure rate was due to differences of double-byte character of our applied Japanese language software for anonymization.

\section{Karger'}




\section{Quantitative Image Analysis}

Three-dimensional models of biliary trees and derived quantitative metrics were obtained from post-processing 3D MRCP acquisitions using proprietary technology (MRCP+ TM software; Perspectum Ltd., Oxford, UK) [2, 3]. The resultant model is a color-coded 3D rendering of the biliary tree with interactive plots showing the variation in diameter along each duct [2]. If ducts had artifacts or unrepresentative portions (e.g., gastrointestinal contamination, artifactual widening near junctions), gaps were introduced in the biliary tree to avoid contamination.

Biliary metrics produced by MRCP+ were (1) number of ducts, (2) number of strictures, (3) number of dilatations, (4) biliary tree volume, (5) total length of ducts, (6) length of strictures or dilatations, (7) duct diameter, and (8) severity of stricture or dilatation (\%). Reference ranges of diameters of healthy bile duct were according to those found by Goldfinger et al. [2] Statistical analysis was not carried out due to the limited number of cases and case reports.

\section{Case Report/Case Presentation}

\section{Reference Cases}

All 3 patient demographics and measured metrics are summarized in Tables 1 and 2, as control. Case 1 shows a representative reference case with a normal biliary tree from a 61-year-old male undergoing cholecystectomy for mild cholecystitis, which underwent cholecystectomy and had no postoperative choledochal complications or alterations (Fig. 1a). However, occult gall bladder cancer was observed and recurred at the remnant cystic duct after 12 months. Case 2 was diagnosed pancreatico-biliary maljunction (PBMJ) without biliary dilatation. MRCP+ showed the dilated duct indicating blue color ( $>8 \mathrm{~mm})$, in which the intrahepatic bile duct was not entirely observed and few strictures were observed. This case underwent the preventable cholecystectomy for occurrence of carcinoma but no remarkable alteration of the remnant common bile duct after surgery. The common hepatic duct shows slightly increased duct diameter, highlighted in green $(>5 \mathrm{~mm})$ and blue-colored portion, indicating a dilatation, at the common hepatic duct (Fig. 1b). Case 3 was diagnosed PBMJ with biliary dilatation and early gallbladder carcinoma, which underwent the extended cholecystectomy and cured after surgery. MRCP+ image showed an increased duct diameter, highlighted in green $(>5 \mathrm{~mm})$ and a blue-colored portion, indicating a dilatation, across the entire extrahepatic bile duct (Fig. 1c). This case was died of other cancer recurrence. All cases underwent cholecystectomy and duration between primary finding and taking MRI was various. The latter 2 cases did not show increased number of strictures or dilatations of the intrahepatic ducts in comparison with that of case 1.

\section{Intrahepatic Biliary Disease Cases}

All 5 patient demographics and measured metrics are summarized in table 1 and 2 . Diseases included 4 cases of hepatolithiasis and a benign severe stenosis of the biliary tree in the left lateral section. Case 4 with hepatolithiasis underwent left hepatectomy and the slightly obstructive jaundice was observed before surgery. In this case, intrahepatic biliary strictures (IHBS) was observed by a radiographer in 6 ducts (arrows) but the number automatically analyzed by MRCP+ was 2 (Fig. 2a), which suggested that the number of IHBS detected by $\mathrm{MRCP}+$ tended to be less than gross findings by the author's detection in this case. Case 5 with hepatolithiasis underwent left hepatectomy. Case 6 with hepatolithiasis underwent left lateral sectionectomy. Case 7 with a stricture by isolated cholangitis underwent left hepatectomy. Case 8 with hepatolithiasis underwent left hepatectomy. All patients had long survivals without occurrence of remnant bile duct cancer. The duration between primary

\section{Karger's}



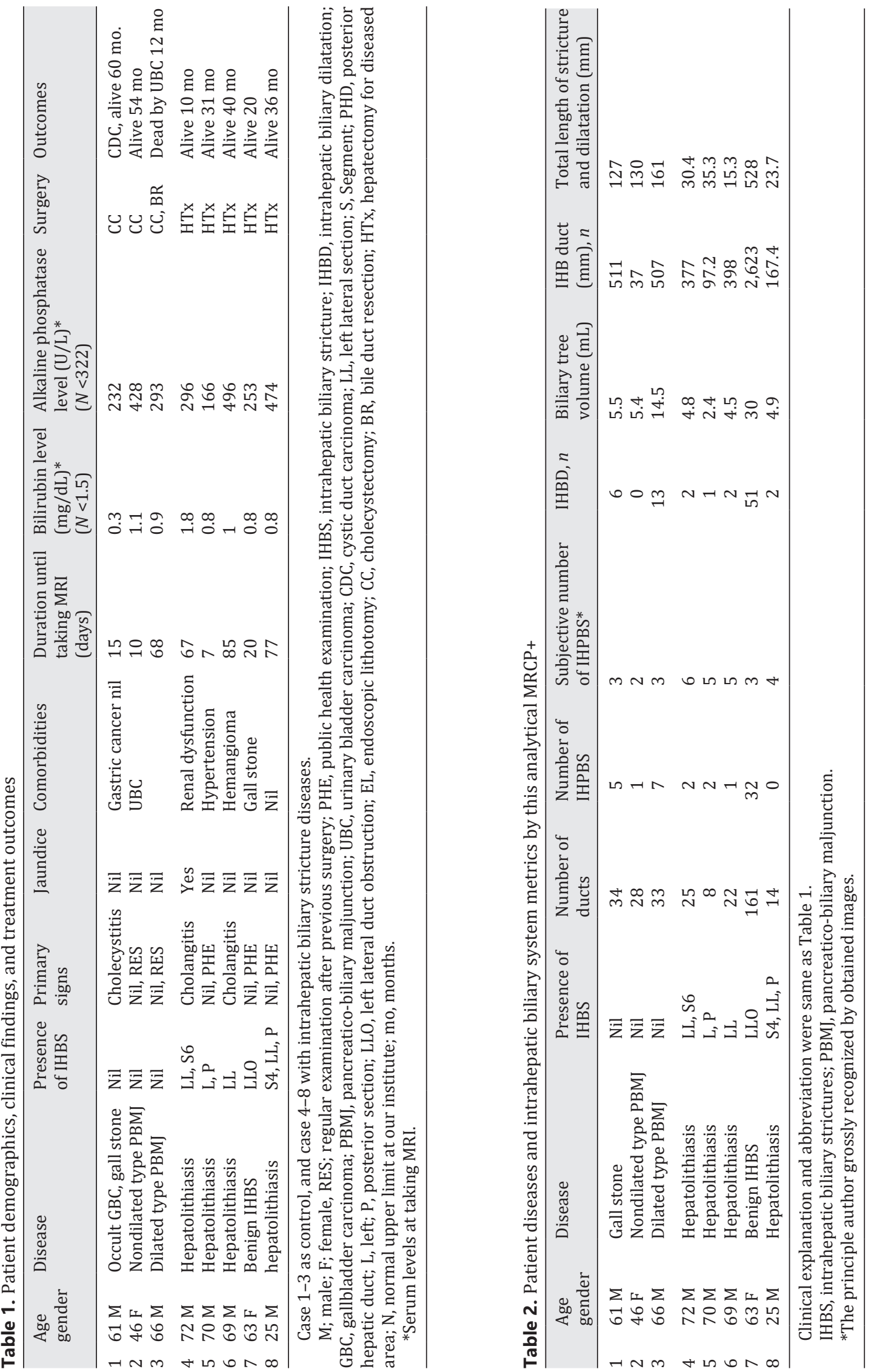
Fig. 1. a A representative case 1 of normal reference where the dilated duct was not entirely observed. b A case 2 diagnosed as of PBMJ without biliary dilatation showing mild increased duct diameter at the common hepatic duct. c A case 3 diagnosed as PBMJ with biliary dilatation and early GBC showing more remarkable dilated duct at the peri-hepatic duct. GBC, gallbladder carcinoma; PBMJ, pancreatico-biliary maljunction.

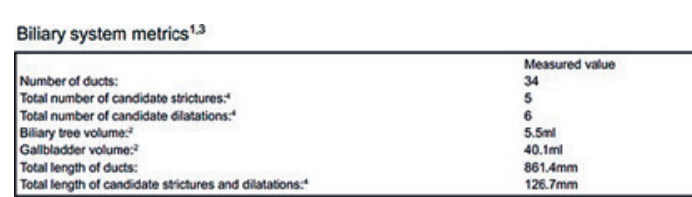

Quantitative biliary tree model

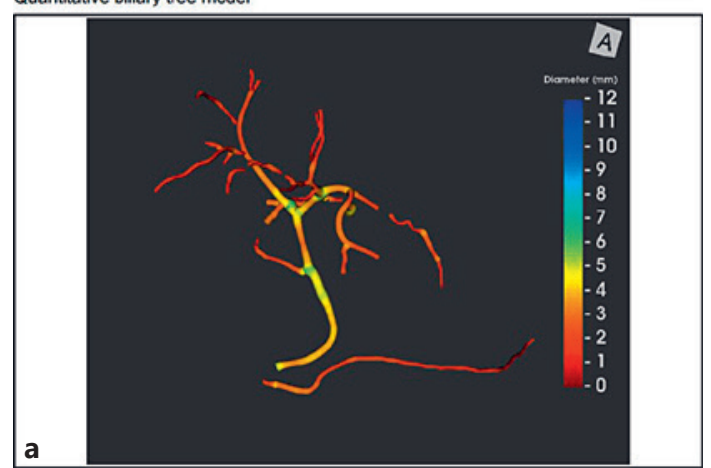

Biliary system metrics ${ }^{1,3}$

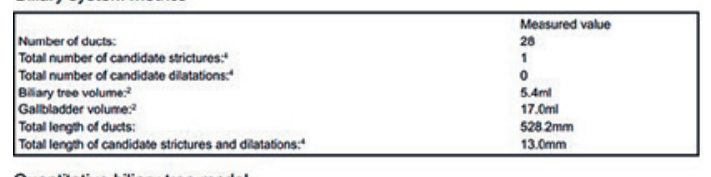

Quantitative biliary tree model

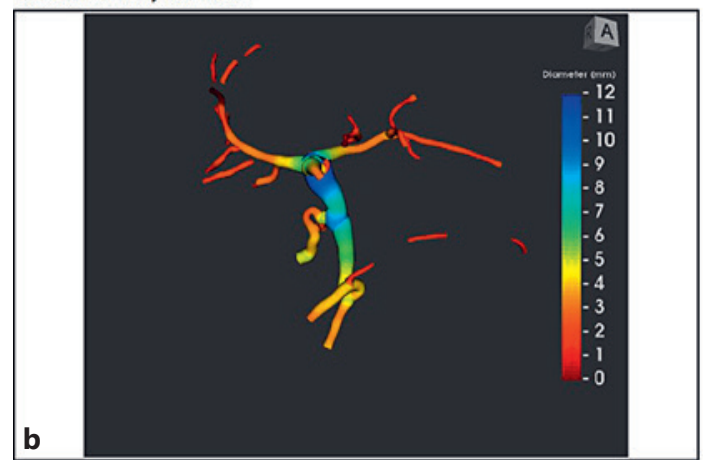

Biliary system metrics ${ }^{1,3}$

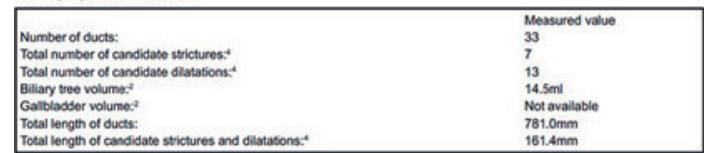

Total length of candidate stictures and dilatatons:

Quantitative biliary tree model

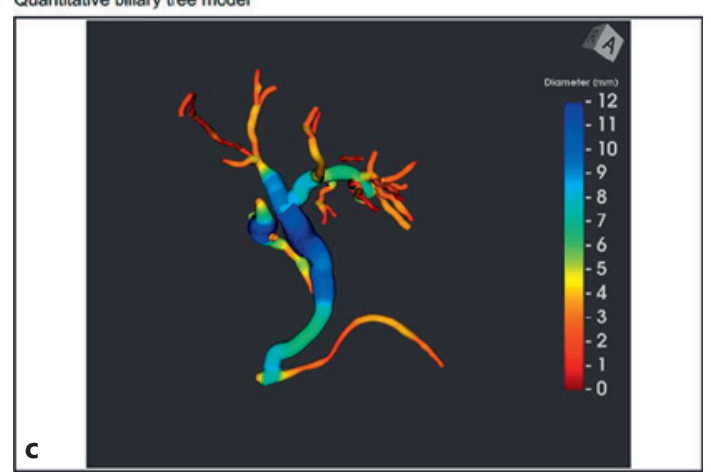


Biliary system metrics ${ }^{1,3}$

\begin{tabular}{|ll|}
\hline & Measured value \\
Number of ducts: & 25 \\
Total number of candidate strictures: $^{4}$ & 2 \\
Total number of candidate dilatations: $^{4}$ & 2 \\
Biliary tree volume: ${ }^{2}$ & $4.8 \mathrm{ml}$ \\
Gallbladder volume: $^{2}$ & Not available \\
Total length of ducts: & $519.7 \mathrm{~mm}$ \\
Total length of candidate strictures and dilatations: & $30.4 \mathrm{~mm}$ \\
\hline
\end{tabular}

\section{Quantitative biliary tree model}

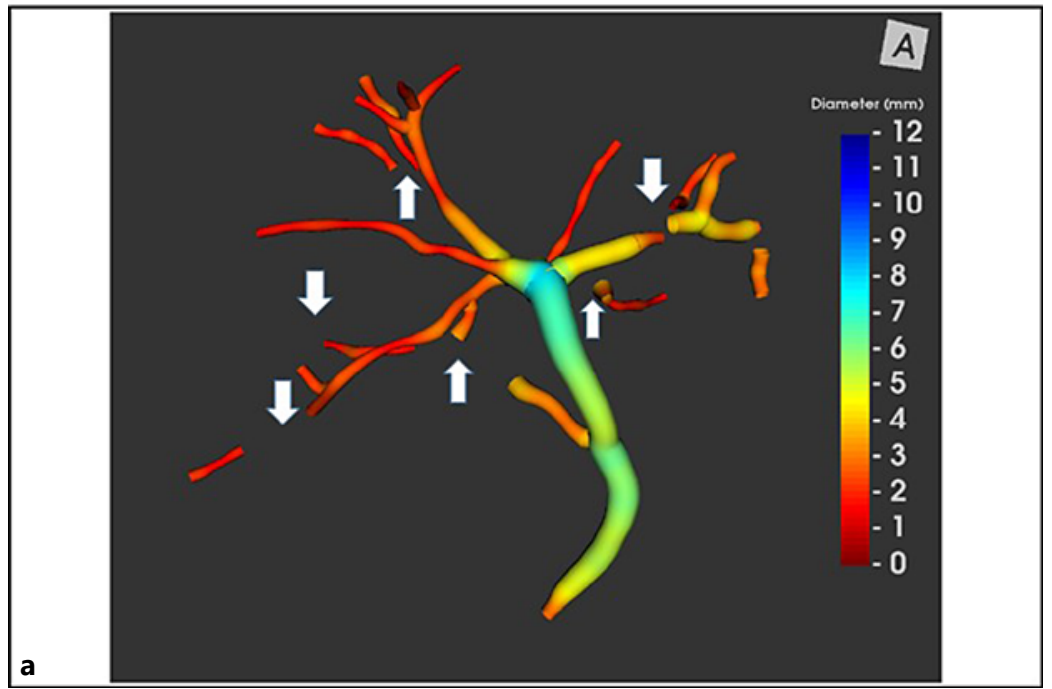

Biliary system metrics ${ }^{1,3}$

\section{Number of ducts:}

Total number of candidate strictures: ${ }^{4}$

Total number of candidate dilatations.

Biliary tree volume: ${ }^{2}$

Gallbladder volume:

Total length of ducts:

Total length of candidate strictures and dilatations:

Measured value

161

32

51

$30.0 \mathrm{ml}$

Not available

$2748.6 \mathrm{~mm}$

\section{Quantitative biliary tree model}

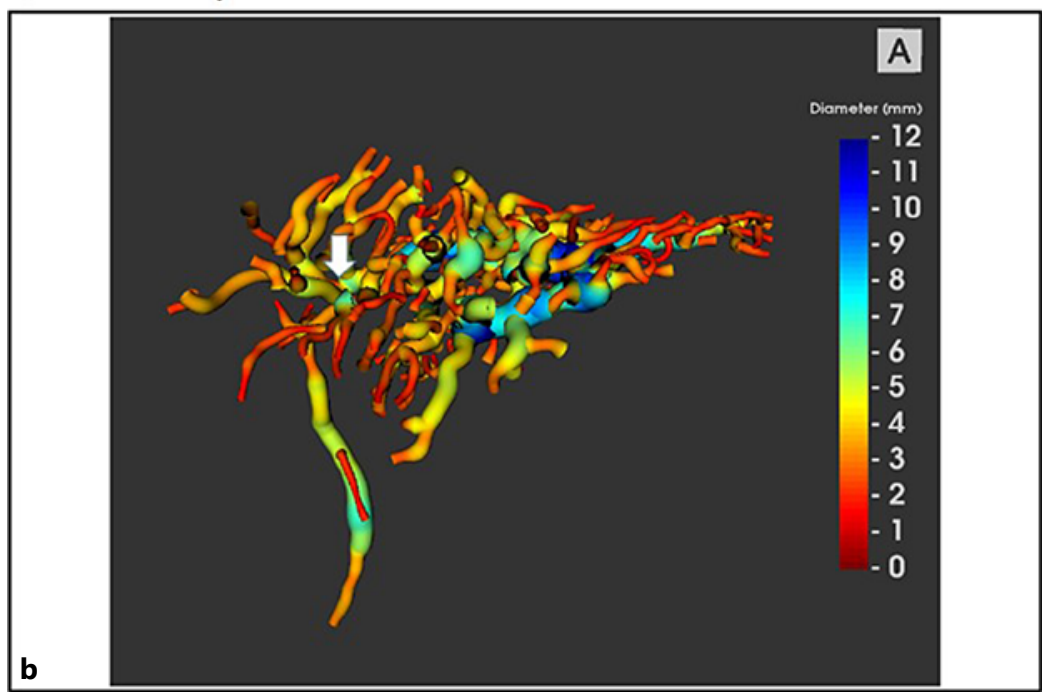

Fig. 2. The representative cases with intrahepatic biliary duct stricture. a Case 4 with hepatolithiasis at left lateral duct and segment 6 ducts, and (b) case 7 with benign intrahepatic biliary stricture due to fibrotic cholangitis indicating peripheral dilated ducts. 
finding and taking MRI, or preoperative clinical findings was various. In this case, biliary obstruction was accompanied by significant peripheral duct dilatation and all MRCP+ metrics were increased (Fig. 2b).

In the summarized examinations by MRCP+, except case 7 , the number of visualized intrahepatic bile ducts was less than 25 , and when compared to the reference cases, the number of intrahepatic biliary strictures or dilatations was not increased. Except case 7, the volume of biliary tree was found to be less than $5 \mathrm{~mL}$. Except case 7, although there was no trend was observed for the total length of intrahepatic biliary duct, the total length of stricture and dilatation was $<100 \mathrm{~mm}$.

\section{Discussion/Conclusion}

MRCP has been advanced for use not only as a diagnostic tool but also as a tool to quantify physiological functions or tissue components. MRCP also has the advantage of not requiring the radiation exposure typically required for other techniques, such as computed tomography $[1,4]$. Thus, MRCP is acceptable for screening and follow-up in patients with biliary diseases with stricture or associated dilatation. The localized alteration of diameter or wall thickening of the bile duct is indicative of precancerous or malignant lesion. When such a disease is diagnosed, further endoscopic biliary investigation at the suspicious lesions is used to define the disease indication and carry out any operative procedures [5]. However, it is relatively difficult to determine the definitive diagnosis in intrahepatic or perihilar bile duct diseases in comparison to those of distal biliary stricture disease due to the late onset of clinical symptoms. We hypothesize that the quantitative metrics obtained from MRCP+ would have higher diagnostic accuracy than diagnosis by radiological physicians [2,3].

Goldfinger et al. and Gilligan et al. [2,3] recently reported the detection of differentiation or alteration of bile duct diameter by 3D colored visualization and associated 2D diagram by a novel artificial intelligence-based algorithm. By their analysis, we could obtain samples of intrahepatic biliary diseases in the present series. To analyze MRCPs using MRCP+, it was necessary to match the MR image reading software between institutions. However, Japanese-based characters used by the reading software at Miyazaki University Hospital found to be incompatible with the MRCP+ post-processing software. Thus, only 8 cases with benign biliary strictures were examined in the present series. In comparison to the 3 reference cases without intrahepatic biliary stricture, the biliary tree volume and the total length of stricture and dilatation metrics tended to be decreased in 5 cases with benign biliary strictures. However, the same trend was not observed for the case with severe stenosis. The number of strictures detected by MRCP+ was found to be lower than that observed by the radiologist. The advantage of automatically visualized diameters of the entire biliary tract is that it confirms localized regions of strictures in cases of intrahepatic biliary stricture diseases at a glance. By assessing the quantitative metrics presented in this analysis in a larger patient cohort, we anticipate MRCP+ will be able to detect the subtle intraluminal irregularities in inter-hepatic biliary diseases [2].

A novel diagnostic tool to determine biliary intraluminal diameter MRCP+ (Perspectum Ltd., Oxford, UK) was examined as a pilot investigation. MRCP+ results in a colored visualization of the entire biliary tract which highlights the diseased parts. However, the number of modeled strictures was found to be lower than that observed by a radiologist. The present analyzed metrics of volume or total length of stricture and dilatation was decreased in 4 of 5 cases with IHBS in comparison with that in 3 cases without strictures. A future prospective study on a larger patient cohort, accompanied with matching of analytical reading software, may show the utility of MRCP+ for detecting biliary diseases at an earlier stage.

This manuscript does not include any nonauthor contributors to acknowledge.

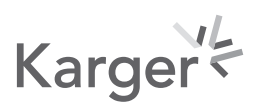




\section{Statements}

All articles must contain the following statements after the main body of the text and before the reference list.

\section{Statement of Ethics}

This study protocol was reviewed and approved by the Clinical Research Support Center, University of Miyazaki, Faculty of Medicine, and approval number \#0 - 0835, November 9, 2020 , and \#0 - 799, October 15, 2020. Written informed consent for publication attached in the submission form was obtained from all 8 patients in each, but no disclaimer was observed.

\section{Conflict of Interest Statement}

The authors have no conflicts of interest to declare.

\section{Funding Sources}

There were no financial supports in this study.

\section{Author Contributions}

Atsushi Nanashima contributed to this study and had first authorship and takes primary responsibility for the original draft. All the authors provided critical revisions to the manuscript. The following is a list of individual author contributions: Atsushi Nanashima, MD, FACS: conceptualization, investigation, management, writing - original draft, and writing review and edit. Masanori Komi, MSc: conceptualization for imaging, preparing images, and investigation. Marija Mavar, MSc: imaging solutions lead. Carlos Ferreira, PhD: MRCP analyst. Paul O’Donoghue, BSc: clinical project manager. Marc Goldfinger, PhD: product specialist and conceptualization. Caitlin Langford, PhD: clinical scientist, reviewing, and English editing. Naoya Imamura, MD: patient follow-up, data collection, and financial support by the Clinical Research Support Expenses of the University of Miyazaki, Faculty of Medicine.

\section{Data Availability Statement}

All data generated or analyzed during this case report are included in this article [and/ or] its see online suppl. material files; for all online suppl. material, see www.karger.com/ doi/10.1159/000518020. Further enquiries can be directed to the corresponding author.

\section{References}

1 Arrivé L, Hodoul M, Arbache A, Slavikova-Boucher L, Menu Y, El Mouhadi S. Magnetic resonance cholangiography: current and future perspectives. Clin Res Hepatol Gastroenterol. 2015 Dec;39(6):659-64.

2 Goldfinger MH, Ridgway GR, Ferreira C, Langford CR, Cheng L, Kazimianec A, et al. Quantitative MRCP imaging: accuracy, repeatability, reproducibility, and cohort-derived normative ranges. J Magn Reson Imaging. 2020 Sep;52(3):807-20.

\section{Karger's}


3 Gilligan LA, Trout AT, Lam S, Singh R, Tkach JA, Serai SD, et al. Diffe rentiating pediatric autoimmune liver diseases by quantitative magnetic resonance cholangiopancreatography. Abdom Radiol. 2020 Jan;45(1):16876.

4 Petitclerc L, Sebastiani G, Gilbert G, Cloutier G, Tang A. Liver fibrosis: review of current imaging and MRI quantification techniques. J Magn Reson Imaging. 2017 May;45(5):1276-95.

5 Nagino M, Hirano S, Yoshitomi H, Aoki T, Uesaka K, Unno M, etal. Clinical practice guidelines for the management of biliary tract cancers 2019: the 3rd English edition. J Hepatobiliary Pancreat Sci. 2021 Jan;28(1):26-54. 